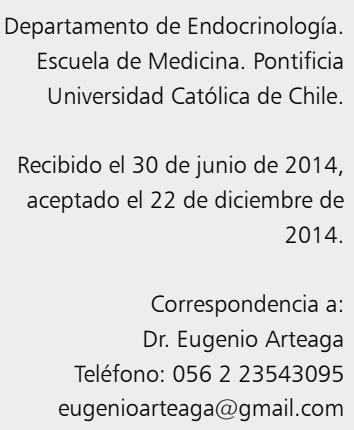

Departamento de Endocrinología. Escuela de Medicina. Pontificia

Recibido el 30 de junio de 2014, 2014

Correspondencia a: Dr. Eugenio Arteaga eugenioarteaga@gmail.com

E 1 hiperparatiroidismo primario ( $\left.\mathrm{HPT} 1^{\circ}\right)$ es una patología frecuente (22-64 casos $\mathrm{x}$ 100.000 habitantes/año) y cuya forma de presentación más prevalente en la actualidad es la asintomática $(80 \%)^{1,2}$. Nuestro objetivo es presentar un caso muy inusual de hiperparatiroidismo primario debido a un macroadenoma paratiroideo palpable, de larga evolución y discutir las posibilidades diagnósticas en casos como el presente. neoplasms.

\section{Macroadenoma cervical palpable. Una presentación muy inusual de hiperparatiroidismo primario}

\author{
ANTONIO ZAPATA P., JOSÉ DELGADO F., \\ GILBERTO GONZÁLEZ V., EUGENIO ARTEAGA U.
}

\section{Cervical macroadenoma causing hyperparathyroidism. Report of one case}

\begin{abstract}
We report a 59-year-old man with a history of hypertension, recurrent renal stones and a severe hypercalcemia of $14.9 \mathrm{mg} / \mathrm{dl}$ with a serum phosphorus of 2.4 $\mathrm{mg} / \mathrm{dl}$ and a serum albumin of $3.6 \mathrm{~g} / \mathrm{dl}$. Physical examination showed a $4 \mathrm{~cm}$ left cervical nodule, consistent with the diagnosis of thyroid nodule. Parathyroid hormone (PTH) levels were $844 \mathrm{pg} / \mathrm{mL}$ (normal 15-65 pg/ml) and a cervical ultrasound examination disclosed a solid nodule in the lower left lobe of $40 x$ $30 \times 25 \mathrm{~mm}$, adjacent to the thyroid parenchyma. Abdominal ultrasound revealed bilateral renal stones. Parathyroid scintigraphy showed a high uptake of the left lower parathyroid mass and a bone densitometry showed bone density $t$ scores of -1.2 in the spine, -2.0 in the right femoral neck and -3.5 in the distal radius. A review of his medical record revealed the presence of hypercalcemia for at least 4 years. He was admitted for hydration and administration of $4 \mathrm{mg}$ zoledronic acid iv. At 24 hours, serum calcium dropped to $11.0 \mathrm{mg} / \mathrm{dl}$, and a left thyroid lobectomy was performed including the lower left parathyroid gland. The pathology report showed a $22.6 \mathrm{~g}$ parathyroid adenoma. Intraoperatory PTH descended $>50 \%$, consistent with successful parathyroidectomy. At 7 days after surgery serum calcium was $8.8 \mathrm{mg} / \mathrm{dl}$, phosphorus $2.1 \mathrm{mg} / \mathrm{dl}$, alkaline phosphatase $166 \mathrm{U} / \mathrm{L}$, albumin $3.9 \mathrm{~g} / \mathrm{dL}$, PTH $230 \mathrm{pg} / \mathrm{ml}$ and $25-\mathrm{OH}$ vitamin D 12.4 $\mathrm{ng} / \mathrm{ml}$. This finding was interpreted as secondary hyperparathyroidism due to vitamin D deficiency and "hungry bone", being less likely the presence of residual or metastatic parathyroid tissue. A cholecalciferol load was administered, with significant descent of PTH.
\end{abstract}

(Rev Med Chile 2015; 143: 396-400)

Key words: Hypercalcemia; Hyperparathyroidism, primary; Parathyroid 
Tabla 1. Resumen de exámenes

\begin{tabular}{|c|c|c|c|c|c|c|c|c|c|c|c|c|c|c|}
\hline & & & & $\begin{array}{c}10 \\
\text { con- } \\
\text { sulta }\end{array}$ & & & & $\begin{array}{l}\text { Ciru- } \\
\text { gía }\end{array}$ & & & & $\begin{array}{l}\text { Carga } \\
\text { Vit. D }\end{array}$ & & \\
\hline Fecha & $\begin{array}{c}30 / 7 / \\
09\end{array}$ & $\begin{array}{c}13 / 5 / \\
10\end{array}$ & $\begin{array}{c}14 / 4 / \\
11\end{array}$ & $\begin{array}{c}15 / 4 / \\
13\end{array}$ & $\begin{array}{c}29 / 4 / \\
13\end{array}$ & $\begin{array}{c}2 / 5 / \\
13\end{array}$ & $\begin{array}{c}6 / 5 / \\
13\end{array}$ & $\begin{array}{c}7 / 5 / \\
13\end{array}$ & $\begin{array}{c}7 / 5 / \\
13\end{array}$ & $\begin{array}{c}7 / 5 / \\
13\end{array}$ & $\begin{array}{c}9 / 5 / \\
13\end{array}$ & $\begin{array}{c}17 / 5 / \\
13\end{array}$ & $\begin{array}{c}8 / 8 / \\
13\end{array}$ & $\begin{array}{l}\text { Nov/ } \\
2013\end{array}$ \\
\hline $\begin{array}{l}\text { Albúmina } \\
(3,5-5 \mathrm{~g} / \mathrm{dL})\end{array}$ & 4,1 & 3,8 & 3,7 & 3,6 & & 3,9 & 3,2 & & & & & 3,9 & 3,9 & 3,6 \\
\hline $\begin{array}{l}\text { Calcemia } \\
(8,5-10,5 \mathrm{mg} / \mathrm{dL})\end{array}$ & 13,9 & 13,8 & 13,5 & 14,9 & 13,7 & 14,3 & 13,1 & 11 & & 11,1 & 9,8 & 8,8 & 10,1 & 9,5 \\
\hline $\begin{array}{l}\text { Calcio iónico } \\
(4,6-5,2 \mathrm{mg} / \mathrm{dL})\end{array}$ & & & & & & 7,7 & & & & & & & & \\
\hline $\begin{array}{l}\text { Fósforo } \\
(2,6-4,5 \mathrm{mg} / \mathrm{dL})\end{array}$ & 2,2 & 1,9 & 2,3 & 2,4 & 2,3 & 1,8 & 2,4 & & & & 1,9 & 2,1 & 3,6 & 3,2 \\
\hline $\begin{array}{l}\text { PTH } \\
(15-65 \mathrm{pg} / \mathrm{ml})\end{array}$ & & & & & & 844 & & 916 & 82 & & 16,6 & 230 & 122,6 & 87,5 \\
\hline $\begin{array}{l}\text { Creatinina } \\
(0,7-1,2 \mathrm{mg} / \mathrm{dL})\end{array}$ & 1,4 & 1,4 & 1,4 & & & & 1,49 & & & & & & & \\
\hline $\begin{array}{l}\text { Fosfatasas } \\
\text { Alcalina } \\
\text { (45-115 U/lt) }\end{array}$ & 121 & 127 & 131 & 135 & 125 & 123 & 106 & & & & & 165 & & \\
\hline $\begin{array}{l}\text { Calciuria } \\
\text { (mg/24 h) }\end{array}$ & & & & & 516 & & & & & & & & & 75,7 \\
\hline $\begin{array}{l}25 \mathrm{OH} \mathrm{D} \\
(\mathrm{ng} / \mathrm{ml})\end{array}$ & & & & & & & & & & 12,4 & & & & 20,7 \\
\hline
\end{tabular}

PTH 844 pg/ml (VN 15-65 pg/ml). La ecografía cervical (Figura 1) mostró un nódulo izquierdo inferior de $40 \mathrm{~mm}$ que desplazaba la tiroides hacia la línea media, heterogéneo, consistencia firme y muy vascularizado; la ecografía abdominal reveló litiasis renal bilateral con imagen dominante de $9 \mathrm{~mm}$ a derecha. Se complementó estudio con cintigrama paratiroideo MIBI-Spect (Figura 2), que mostró captación aumentada en paratiroides inferior izquierda. La densitometría ósea de doble fotón radiológico (Lunar Prodigy, score T) mostró L2-L4 -1.3, cuello femoral derecho -2.0, y radio distal -3.5. La revisión de sus exámenes previos mostró hipercalcemia desde al menos 4 años.

Con diagnóstico de hiperparatiroidismo primario de larga evolución con compromiso óseo y renal, se hospitalizó para hidratación y administración de zoledronato $4 \mathrm{mg} \mathrm{IV}$, como preparación para resolución quirúrgica. A las $24 \mathrm{~h}$, habiendo descendido la calcemia a $11 \mathrm{mg} / \mathrm{dL}$, se efectúa lobectomía tiroidea izquierda, que incluía nódulo paratiroideo adherido. La biopsia fue informada como adenoma paratiroideo de $28 \mathrm{~mm}$ y $22,6 \mathrm{~g}$, adherido a lóbulo tiroideo, bien delimitado, con cápsula delgada completa, sin signos de neoplasia maligna en los cortes examinados y sin ganglios comprometidos. La curva de PTH fue sugerente de curación, con caída $>90 \%$ (PTH preoperatorio $916 \mathrm{pg} / \mathrm{ml}$ y $15 \mathrm{~min}$ post extirpación $88,2 \mathrm{pg} / \mathrm{ml})^{3}$.

$\mathrm{Al} 3^{\circ}$ día postoperatorio el paciente evolucionó asintomático con calcemia $9,8 \mathrm{mg} / \mathrm{dL}$; fosfemia $1,9 \mathrm{mg} / \mathrm{dL}$; PTH 16,6 pg/ml, siendo dado de alta. Sin embargo, a los 7 días de la cirugía se observó calcemia 8,8 mg/dL; fosfemia 2,1 mg/dL; FA 166 $\mathrm{U} / \mathrm{L}$; albuminemia 3,9 g/dL, PTH 230 pg/ml y 25OHD 12,4 ng/ml (VN > $20 \mathrm{ng} / \mathrm{ml})$. Se procedió a una carga de colecalciferol (50.000 UI semanal v.o. $\mathrm{x} 6$ veces) y terapia de mantención con carbonato de calcio/vitamina D $500 \mathrm{mg} / 800 \mathrm{UI}$ x 2 veces/día, con lo que se observó a los 6 meses de la cirugía mejoría de $25-\mathrm{OHD}(20,7 \mathrm{ng} / \mathrm{ml})$, descenso de PTH $(87,5$ pg/ml) y mantención de calcemia 

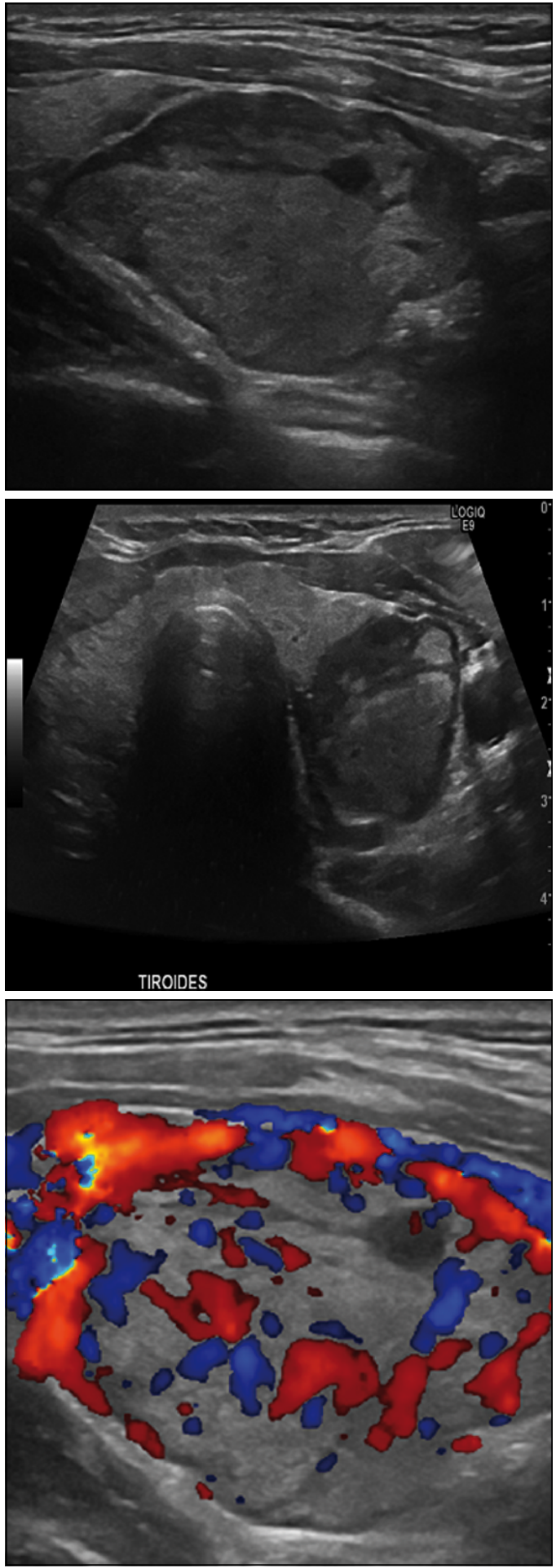

Figura 1. Ecografía Cervical que muestra nódulo izquierdo inferior de 40 × $30 \times 25$ mm que desplazaba la tiroides hacia la línea media, heterogéneo y vascularizado.

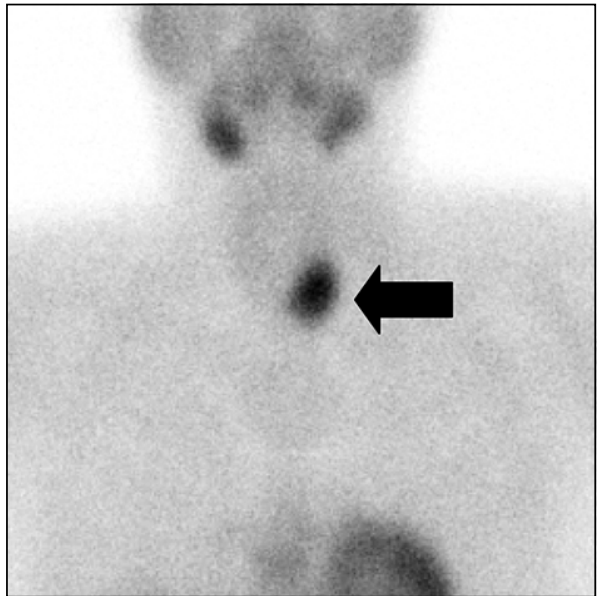

Figura 2. Cintigrama paratiroideo Sesta MIBI-Spect que mostró hipercaptación en relación a paratiroides inferior izquierda.

normal $(9,5 \mathrm{mg} / \mathrm{dL})$. Paciente nota mejoría de bradipsiquia y de su rendimiento intelectual durante la evolución.

\section{Discusión}

El presente caso ilustra una presentación extraordinariamente inusual de hiperparatiroidismo primario, dada la palpación de un nódulo paratiroideo. Esto último sólo se reporta en casos aislados y abre siempre la necesidad de realizar diagnóstico diferencial con cáncer de paratiroides.

El cáncer de paratiroides es una neoplasia poco frecuente $\left(<1 \%\right.$ de los HPT $\left.1^{\circ}\right)$, y suelen ser tumores de gran tamaño, usualmente esporádicos, irregulares, duros y adheridos a planos profundos, similares a la presentación de nuestro paciente. La distinción entre lesiones benignas y malignas es difícil y rara vez se hace en la biopsia inicial. Considerando que los hallazgos histológicos son poco específicos, los criterios a considerar para el diagnóstico definitivo de cáncer de paratiroides son: invasión local o a estructuras contiguas, metástasis linfáticas o a distancia ${ }^{4}$. La mutación en el gen HRPT2, con reducción en la expresión de parafibromina, estaría involucrada en su patogenia y podría ser utilizada como apoyo en caso de duda diagnóstica ${ }^{5}$. Suelen presentarse con niveles de calcemia elevados (>14 mg/dl) y con compromiso sistémico significativo (osteítis fibrosa quística, resorción subperióstica, osteopenia 
difusa, nefrocalcinosis, nefrolitiasis y falla renal, entre otros); la mayoría de las veces el diagnóstico se hace en forma retrospectiva, cuando recurre la hipercalcemia después de la cirugía ${ }^{6}$. En nuestro caso, la presentación con nódulo palpable y compromiso sistémico asociado altos niveles de PTH e hipercalcemia grave hacían plantear cáncer de paratiroides como etiología del HPT $1^{\circ}$ de nuestro paciente; sin embargo, el hallazgo intraoperatorio, el informe de anatomía patológica y la evolución con calcemia normal a los 6 meses de la cirugía, hacen plantear que se trata de una lesión benigna.

Existen pocos reportes respecto a adenomas de gran tamaño. El peso promedio de los adenomas paratiroideos operados se ha estimado en $520 \mathrm{mg}$ en la última década ${ }^{7}$. Un estudio analizó las características de 26 casos con adenomas paratiroideos determinó un peso de $>3,5 \mathrm{~g}$ (promedio de 9,8 g y el mayor de $40 \mathrm{~g}$ ), $50 \%$ de los cuales fueron ectópicos y con un promedio de PTH de $451 \mathrm{pg} /$ $\mathrm{ml}$; un tercio de los casos fueron descubiertos incidentalmente por hipercalcemia, sin reportarse lesiones malignas ${ }^{8}$. Análisis genéticos de los adenomas de $>4 \mathrm{~g}$ han mostrado bajos índices de proliferación, con mutaciones somáticas en el gen de la menina (25\%) y en el gen HRPT2, y alteración de la expresión de parafibromina en $50 \%$ de los casos, lo que podría dar un sustento genético a estas presentaciones más sintomáticas ${ }^{9}$. Además, estudios muestran que a mayor tamaño del adenoma paratiroideo existiría mayor mortalidad ${ }^{10}$. Se ha intentado correlacionar los niveles de PTH preoperatoria con el tamaño y peso del adenoma paratiroideo, pero la asociación ha sido débil ${ }^{11}$.

Respecto al manejo preoperatorio de casos como el presente, existe consenso en la necesidad de hidratar adecuadamente. Sin embargo, no existe acuerdo en relación al uso de bisfosfonatos inyectables. Algunos estudios retrospectivos y series de casos sostienen que el uso de bisfosfonatos en el preoperatorio puede mejorar la hipercalcemia y disminuir el riesgo de hueso hambriento en el post operatorio ${ }^{12,13}$, pero existen casos reportados que muestran un efecto contrario ${ }^{14}$. En nuestro paciente, dado la grave hipercalcemia y la alta probabilidad de desarrollo de hueso hambriento después de la cirugía, decidimos pre tratar con bisfosfonatos. Es necesario estudios prospectivos y de distribución aleatoria que evalúen esta controversia, para definir el beneficio del uso de bisfosfonatos en estos pacientes ${ }^{15}$. Respecto a la vitamina $\mathrm{D}$, es recomendable tener sus niveles adecuados en el preoperatorio (disminuiría el riesgo de hueso hambriento postparatiroidectomía ${ }^{15}$; sin embargo, considerando la urgencia quirúrgica del paciente, no se realizaron cargas preoperatorias para mejorar sus niveles.

Un fenómeno interesante es el rebote de los niveles de PTH después de la cirugía. Si bien, este podría ser un argumento a favor de la malignidad del tumor extirpado y la posibilidad de que hubiera tejido paratiroideo remanente o metastásico, esto parece muy improbable dada la normalización persistente de la calcemia y el descenso de $90 \%$ de la PTH en el pabellón. Nuestra hipótesis es que el alza de PTH a partir del 7o día de la cirugía fue multifactorial: por un lado pudo deberse a cierto grado de hueso hambriento, (manifestado por persistencia de fosfatasas alcalinas elevadas, fósforo bajo y calciuria relativamente baja a pesar del aporte de $1.000 \mathrm{mg}$ de calcio) que probablemente fue atenuado por el efecto del ácido zoledrónico; otro mecanismo potencial es la deficiencia de vitamina $\mathrm{D}$ previa a la cirugía. A favor de esta última hipótesis está el hecho que después de una carga de vitamina $\mathrm{D}$, se asistió a mejoría en los niveles de esta hormona, aunque aún en rangos de insuficiencia (probablemente por suplementación submáxima) y atenuación del hiperparatiroidismo secundario, permaneciendo la calcemia dentro de rangos normales.

Existe asociación entre bajos niveles de vitamina $\mathrm{D}$ y formas más severas de HPT primario ${ }^{16-18}$, siendo los pacientes adultos mayores, sometidos a cirugía bariátrica, gastrectomía, portadores de síndromes de malabsorción y usuarios de anticonvulsivantes las poblaciones, de mayor riesgo de déficit ${ }^{19,20}$. En Chile, un estudio de nuestro grupo mostró que el déficit de vitamina $\mathrm{D}$ es común en ciertas poblaciones específicas, aun con niveles de exposición solar normal ${ }^{21}$.

Otro aspecto a destacar es la ausencia de diagnóstico oportuno en un individuo con hipercalcemia grave documentada durante al menos 4 años, sintomática, y con controles médicos periódicos de salud, que presentaba antecedentes de urolitiasis recurrente y refería bradipsiquia. Parece razonable reforzar en la enseñanza de medicina de pre y post grado que las hipercalcemias, aun siendo leves, requieren ser derivadas a especialistas para estudio adecuado. Además, existe consenso en que todo paciente con litiasis urinaria recurrente debe 
ser estudiado desde el punto de vista metabólico, para precisar la causa e implementar medidas de tratamiento médico preventivo. La persistencia de hiperparatiroidismo primario por muchos años, como ocurrió en el presente caso, no sólo implica riesgos óseos y renales, sino que pone al paciente en alto riesgo de sufrir complicaciones más inusuales como pancreatitis aguda o compromiso de conciencia e incluso estado de coma, si el paciente se complica con un cuadro de deshidratación o es sometido a inmovilidad prolongada.

En resumen, se presenta un caso muy inhabitual de HPT $1^{\circ}$ debido a un adenoma paratiroideo palpable, que evolucionó sin diagnóstico por más de 4 años, a pesar del antecedente de hipercalcemia y urolitiasis recurrente. El ascenso de PTH a la semana de la cirugía se interpretó como hiperparatiroidismo secundario a deficiencia de vitamina D y posiblemente a la acreción ósea que se generó pos cirugía.

\section{Referencias}

1. Khan A, Bilezikian J. Primary hyperparathyroidism: pathophysiology and impact on bone. CMAJ 2000; 163 (2): 184-7.

2. Bahrami A, Montazeri V, Barband V, Barband AR, Poorzand A. Advanced Bone Disease as the most Common Clinical Presentation of Primary Hyperparathyroidism in Irianians and Clinical and Laboratory Features of 62 Patients from North- western Iran. Int J Endocrinol Metab 2006; 4: 19-29.

3. Domínguez JM, Velasco S, Goñi I, León A, González H, Claure R, et al. Utilidad de la PTH intraoperatoria como predictor de curación quirúrgica en hiperparatiroidismo primario. Rev Med Chile 2009; 137: 1591-7.

4. El-Hajj G, Arnold A. Parathyroid Carcinoma. En Upto Date on line, [revisado en junio de 2014].

5. Marconni C, Cetani F, Rubin M, Silverberg S, Piachera A, Bilezikian J. Parathyroid carcinoma. J Bone Miner Res 2008; 23 (12): 1869-80.

6. Shane E. Clinical Review 122: Parathyroid carcinoma. J Clin Endocrinol Metab 2001; 86 (2): 485-93.

7. Almquist $M$, Bergenfelz A, Martensson $H$, Thier M, Nosdenstrom E. Changing biochemical presentation of primary hyperparathyroidism. Langenbecks Arch Surg 2010; 395 (7): 925-8.

8. Lalanne-Mistrih ML, Ognois-Ausse P, Goudet P, Cougard P. Giant parathyroid tumor: characterization of gland weighing more than 3,5 grams. Ann Chir 2002;
127 (3): 198-202.

9. Sulaiman L, NilssonIL, Juhlin CC. Genetic characterization of large parathyroid adenomas. Endocr Relat Cancer 2012; 19 (3): 389-407.

10. Hedback G, Oden A, Tisell LE. Parathyroid adenoma weight and the risk of death after treatment for primary hyperparathyroidism. Surgery 1995; 117 (2): 134-9.

11. Goñi I, Manzor M, León A, Solar A, Ruiz C. Correlación entre el nivel preoperatorio de paratohormona y el volumen y peso del adenoma de glándula paratiroides extirpado, en pacientes con hiperparatiroidismo primario mejorados. Rev Chil Cir 2014; 66 (1): 56-92.

12. Davenport A, Stearn MP. Administration of pamidronate helps prevent inmediate postparathyroidectomy hungry bone síndrome. Nephrology 2007; 12 (4): 386-90.

13. Lee I, Sheu W, Tu S, Kuo S, Pei D. Bisphosphonate pretreatment attenuates hungry bone syndrome postoperatively in subject with primary hyperparathyroidism. J Bone Miner Metab 2006; 24 (3): 255-8.

14. Corsello S, Paragliola R, Locantore P, Ingraudo F, Ricciato $\mathrm{M}$, Rota C, et al. Post-surgery severe hipocalcemia in primary hyperparathyroidism preoperatively treated with zolendronic acid. Hormones 2010; 9 (4): 338-42.

15. Witeveen JE, van Thiel S, Romijn JA, Hamdy NA. Hungry Bone Syndrome: still a challenge in the postoperative managenment of primary hyperparathyroidism: a systematic review of the literature. Eur J Endocrinol 2013; 168 (3): R45-R53.

16. Moosgaard B, Vestergaard P, Heickendorff L, Melsen F, Christiansen P, Mosekilde L. Vitamin D status, seasonal variations, parathyroid adenoma weight and bone mineral density in Primary Hyperparathyroidism. Clin Endocrinol 2005; 63 (5): 506-13.

17. Souberbielle J, Maury E, Friedlander G, Cormier C. Vitamin D and Primary Hyperparathyroidism. J Steroid Biochem Mol Biol 2010; 121 (1-2): 199-203.

18. Silberberg S, Shane E, Dempster D, Bilezikian J. The effects of vitamin D insufficiency in patients with Primary Hyperparathyroidism. Am J Med 1999; 107 (6): 561-7.

19. Miranda D, Leivga L, León J, De la Maza M. Diagnóstico y Tratamiento de la Deficiencia de Vitamina D. Rev Chil Nutr 2009; 36 (3): 269-77.

20. Agarwal A, Kumpar S, Sukumar R. Hyperparathyroidism and Malnutrition with severe vitamin D deficiency. World J Surg 2009; 33: 2303-13.

21. González G, Alvarado JN, Rojas A, Navarrete C, Velásquez CG, Arteaga E. High prevalence of vitamin D deficiency in Chilean healthy postmenopausal women with normal sun exposure: Additional evidence for a worldwide concern. Menopause 2007; 14 (3): 455-61. 